\section{Observation of new vibronic luminescence band in semiconducting diamond}

\section{J.A. Freitas, Jr., P.B. Klein and A.T. Collins}

Indexing terms: Photoluminescence, Semiconductor materials

Steady state and time-resolved photoluminescence studies have been carried out for a new vibronic emission band, with a zero phonon line at $2.395 \mathrm{eV}$, that is observed in type IIb semiconducting diamond. This band exhibits a relatively slow (228ns) photoluminescence decay that is exponential, and is believed to be due to an internal transition at a defect site

While the optical properties of natural diamond have been investigated for many years [1], recent advances in the growth of high quality diamond films by chemical vapour deposition (CVD) [2] have generated a greatly renewed interest in this material for the development of electronic devices for use in adverse conditions. The optical characterisation of these CVD films has relied heavily on the wealth of information that exists from studies of bulk natural and high pressure high temperature (HPHT) synthesised diamonds.

We report the observation of a new vibronic emission band in an HPHT semiconducting diamond (type IIb: boron-doped). Given that diamond has been studied optically for more than four decades, with scores of emission and absorption bands identified, the observation of a new band is relatively unexpected. We believe that our observation of a new emission band may be due to the fact that there have not been very many photoluminescence (PL) studies carried out in bulk semiconducting diamond with exciting photon energies that are well below the $\sim 5.5 \mathrm{eV}$ indirect gap. It has been known for some time that in III-V compounds, for example, such excitation conditions are ideal for probing emission bands that involve deep levels or internal transitions [3]. In the present case, we believe that above- or near-gap excitation created by photons (PL) or electrons (cathodoluminescence, $\mathrm{CL}$ ) creates a situation whereby some emission bands which can be excited efficiently by excitation near midgap cannot compete effectively with the more dominant, and consequently more well-known emissions, and have thus gone unobserved.

The present measurements have been carried out under both steady state and pulsed excitation. The $\mathrm{CW}$ measurements employed both visible and UV emission lines of an argon ion laser, and were carried out at $6 \mathrm{~K}$. Time-resolved measurements were performed at $10 \mathrm{~K}$ with the $355 \mathrm{~nm}$ frequency-tripled output of a 10ns Q-switched YAG laser. Two boron doped HPHT synthetic samples were studied. Sample 1 was grown using $\mathrm{Ni}$ as a solvent-catalyst, whereas for sample $2 \mathrm{Fe}-\mathrm{Al}$ was employed. $\mathrm{Al}$ was used to getter nitrogen $(\mathrm{N})$, thus lowering the concentration of $\mathrm{N}$ and increasing the concentration of neutral boron acceptors through diminished compensation.

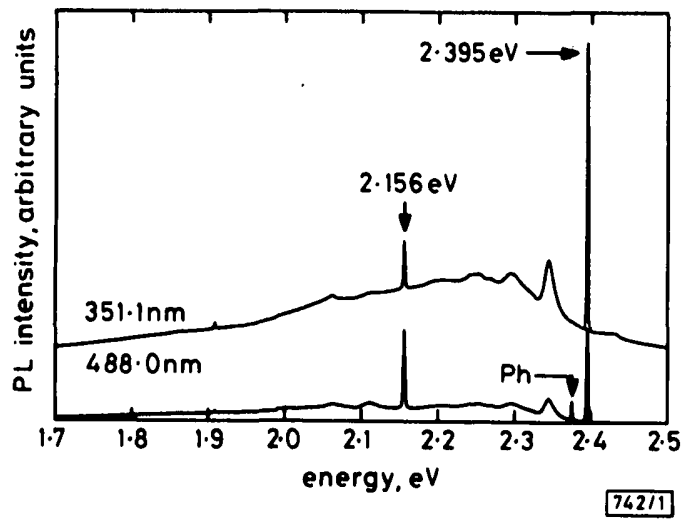

Fig. 1 CW PL spectra from sample 2, excited with 351.1 and $488.0 \mathrm{~nm}$ laser lines

The new vibronic band at $2.395 \mathrm{eV}$ is more efficiently excited at 351.1 $\mathrm{nm}$. The broad green emission band is not efficiently excited at $\mathbf{4 8 8 . 0}$ $\mathrm{nm}$. $\mathrm{Ph}$ indicates the first order Raman phonon line of diamond.

Fig. 1 shows CW spectra from sample 2 in the range 1.7-2.5eV.
In both spectra represented in Fig. 1 we observed the $2.156 \mathrm{eV}$ vibronic band associated with $\mathrm{N}$-vacancy complexes [4]. The $\mathrm{CW}$ spectra also exhibit a new vibronic system with a zero-phonon line (ZPL) at $2.395 \mathrm{eV}$, and with the main phonon replicas spaced $\sim 51$ $\mathrm{meV}$ apart. To our knowledge, this band has not previously been reported, because the $\mathrm{ZPL}(2.395 \mathrm{eV})$ and the main phonon replicas $(\sim 51 \mathrm{meV})$ do not appear to match any of the vibronic bands appearing in the literature. Note that both sets of vibronic bands are superimposed on a broad green emission band at $\sim 2.2 \mathrm{eV}$ [5] that is observed when excited at $351.1 \mathrm{~nm}$, but not under $488.0 \mathrm{~nm}$ excitation. The dependence of the relative intensities of the 2.156 and $2.395 \mathrm{eV}$ bands on excitation wavelength was found to be consistent with a small lattice distortion (i.e. symmetric absorption and emission spectra) of both bands. Absorption measurements are presently under way to confirm this. Under electron excitation (CL), emission in the region of the green band totally dominates that of the $2.395 \mathrm{eV}$ band [5]. This, taken with the above results, tends to suggest that excitation near or above the band edge excites efficient processes that dominate the $2.395 \mathrm{eV}$ emission, whereas at much lower excitation energies this band is easily seen, as the competing processes are much less important in comparison.

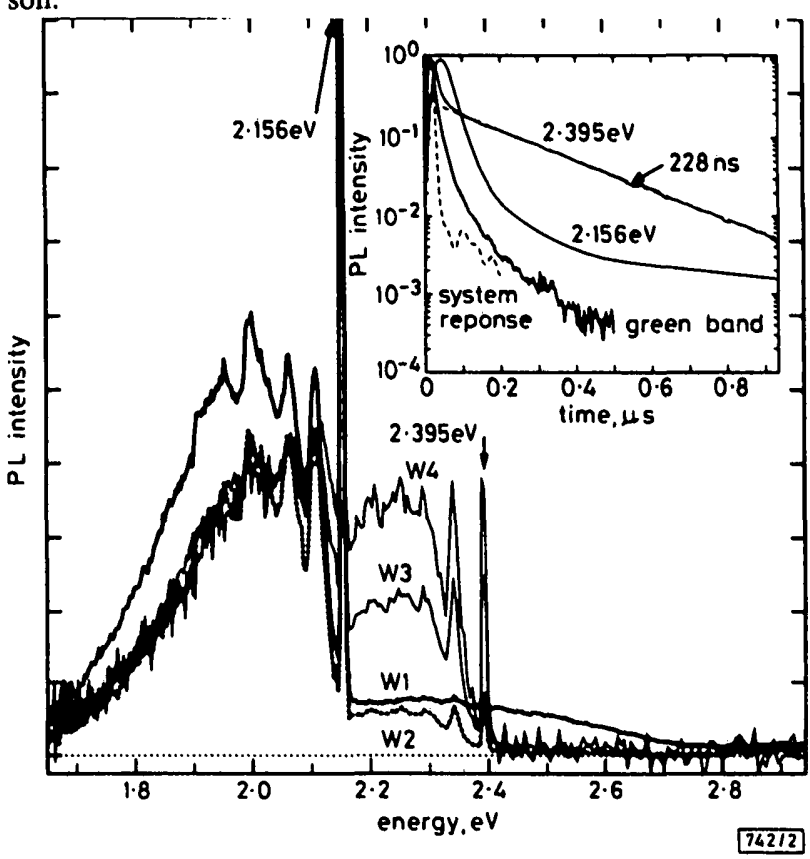

Fig. 2 Time resolved spectra of diamond sample 2 at 10K, excited at $354.7 \mathrm{~nm}$, for four time windows relative to start of excitation pulse

The spectra are all normalised at $2.156 \mathrm{eV}$, so that the slower $2.395 \mathrm{eV}$ system appears more intense for later time windows.

Inset: exponential PL decay of the $2.395 \mathrm{eV}$ band, which is compared to the much faster, nonexponential behaviour of the $2.156 \mathrm{eV}$ band anc the $2.2 \mathrm{eV}$ green emission.

$\mathrm{W} 1=0.5 \mathrm{~ns}$

$\mathrm{W} 2=50-100 \mathrm{~ns}$

$\mathrm{W3}=100-200 \mathrm{~ns}$

W4 $=200-500 \mathrm{n}$

Similar spectra are observed for this sample under pulsed excitation in the time-resolved data shown in Fig. 2. The spectra are all normalised at the $2.156 \mathrm{eV}$ peak energy, so that the slower $2.395 \mathrm{eV}$ system appears increasing in intensity for later time windows. At early times (0-50ns time window) the spectrum is dominated by the $2.156 \mathrm{eV}$ system, while the $2.395 \mathrm{eV}$ band appears as a minor feature on the broad green $2.2 \mathrm{eV}$ emission band. At later times, however, the short-lived green band disappears, leaving prominent only the $2.156 \mathrm{eV}$ and the new $2.395 \mathrm{eV}$ systems. The spectra taken during later time windows indicate that the $2.156 \mathrm{eV}$ system is decaying more rapidly than the $2.395 \mathrm{eV}$ system. This can be seen more clearly in the inset in Fig. 2, where PL decays are compared for the green emission as well as the 2.156 and $2.395 \mathrm{eV}$ systems. Both the green band and the $2.156 \mathrm{eV}$ system exhibit a non-exponential decay: the initial component of the 
$2.156 \mathrm{eV}$ band decay is consistent with the literature value of $29 \mathrm{~ns}$ [4]. In contrast, the $2.395 \mathrm{eV}$ decay exhibits a sharp decrease at early times, which is associated with the fast decay of the broad green band background (see Fig. 2), followed at later times by the decay of the $2.395 \mathrm{eV} \mathrm{ZPL}$ itself, which we have observed to be accurately exponential for well over two orders of magnitude (not evident in Fig. 2). The 228ns exponential decay time is relatively long for a vibronic band in diamond, as most of the reported decay times of defect emissions in diamond are a few to tens of nanoseconds[6]. The enhanced intensity of the $2.156 \mathrm{eV}$ phonon sidebands in the $0-50 \mathrm{~ns}$ time window is the result of contributions from both the broad green band as well as a broad red emission that has been reported recently [5].

At present, a precise identification of the origin of the $2.395 \mathrm{eV}$ band is not possible. However, from the exponential nature of the decay it can be concluded that this emission band most likely results from an internal transition associated with a point defect or complex centre, and that there are no efficient recombination pathways to compete with the emission under these excitation conditions. The latter may not be the case under above-gap excitation. While the $2.395 \mathrm{eV}$ system is observed quite easily in sample 2 , it is not observed in sample 1 , and it is seen to be very weak in two natural type IIb diamonds. The presence of this band seems to correlate with higher concentrations of uncompensated boron acceptors. It is thus tempting to identify this band with a complex involving boron, but measurements on a wider range of specimens will be necessary to confirm this interpretation.

Electronics Letters Online No: 19931071

13 July 1993

J. A. Freitas, Jr. (Sachs-Freeman Associates, Landover MD 207855396, USA)

P. B. Klein (Naval Research Laboratory, Washington DC 20375-5347, USA)

A. T. Collins (Department of Physics, Kings College London, Strand, London WCR2R 2LS, United Kingdom)

\section{References}

1 WALKER, J.: 'Optical absorption and luminescence in diamond', Rep. Prog. Phys., 1979, 42, pp. 1605-1659

2 SNAIL, K.: 'Growth, processing and properties of CVD diamond for optical applications', Opt. Mater., 1992, 1, pp. 235-258

3 TAJIMA, M.: 'Deep level photoluminescence in GaAs', in J. CHIKAWA, $K$. SUMINo and K. WADA (Eds.): Defects and properties of semiconductors: Defect engineering, (KTK Scientific Publications, Tokyo, 1987), p.37-69

4 DAviEs, G.: 'The Jahn-Teller effect and vibronic coupling at deep levels in diamond', Rep. Prog. Phys., 1981, 44, pp. 787-830

5 FREITAS, JR. J.A., STROM, U., and COLLINS, A.T.: 'Photoluminescence and cathodoluminescence studies of semiconducting diamond', Diamond and Related Mater., 1993, 2, pp. 87-91

6 DAVIES, G.: 'The optical properties of diamond', in WALKER P. L., Jr., and THROWERP. A. (Eds.): 'Chemistry and physics of carbon. Vol. 131' (Marcel Dekker, New York, 1977), pp.1-143

\section{High temperature superconductor lumped element resonator}

\author{
M.J. Lancaster, J.C. Li, A. Porch and N.G. Chew
}

Indexing terms: Resonators, High-temperature superconductors

The authors have studied a high temperature superconducting (HTS) lumped element resonator consisting of an inductance and capacitance in parallel. It has a low power unloaded quality factor $Q_{0}$ in excess of 9000 at $5.7 \mathrm{GHz}$ and $1 \mathrm{~K}$. The resonator is just over $1 \mathrm{~mm}$ square significantly smaller than any other device with comparable $\mathrm{Q}$ at this centre frequency. The authors also investigate the implementation of the resonator in a bandstop filter.

Introduction: The aim of these designs and investigations is tc demonstrate the application of planar lumped elements in the gigahertz range and the effect of using HTS material, in this case thin films of $\mathrm{YBa}_{2} \mathrm{Cu}_{3} \mathrm{O}_{7}$ (YBCO) on $\mathrm{MgO}$ substrates. Lumped element devices, with dimensions smaller than a wavelength, can offer considerable size-saving while still maintaining the performance of equivalent larger devices made using normal metal. The use of a coplanar structure has also made mounting and fabrication easier because only one side of the film requires deposition. Details of the film deposition and device packaging are given in [1].
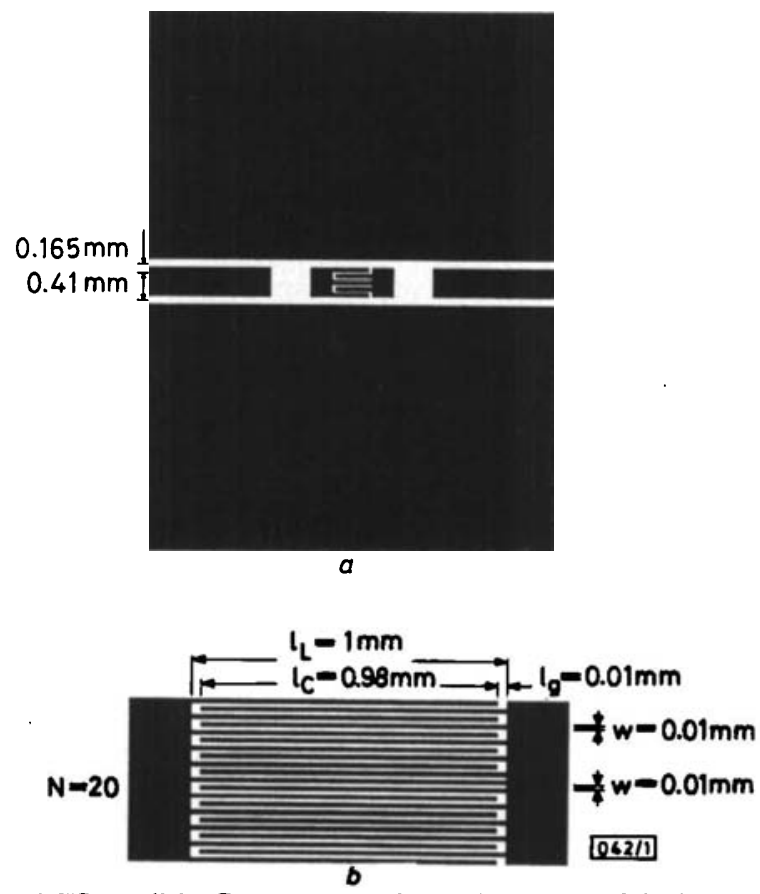

Fig. 1 HTS parallel LC resonator and an enlargement of the lumped elements (not to scale)

a HTS parallel LC resonator

b Enlargement of lumped elements

Parallel LC resonator: The resonator is shown in Fig. 1. Fig. la shows how the resonator is mounted with two $50 \Omega$ feed lines and coupling gaps. Fig. $1 \mathrm{~b}$ shows an enlargement of the resonator element. It consists of 20 interdigital capacitor fingers, with the middle one shorted as an inductor. The dimensions of the device are also shown in Fig. 1. The choice of large coupling gaps of $1 \mathrm{~mm}$ on either side is deliberate because we want to isolate the resonator from the $50 \Omega$ feed lines in order to measure the unloaded $Q$ with very little correction.

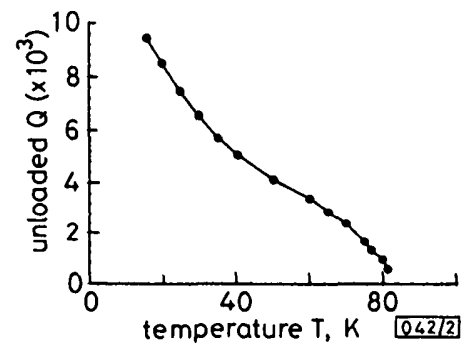

Fig. 2 Unloaded quality factor of resonator as function of temperature

Fig. 2 illustrates the unloaded quality factor $Q_{0}(T)$ as a function of temperature at input power of $-10 \mathrm{dBm}$. It ranges from 9400 at $5.734 \mathrm{GHz}$ and $15 \mathrm{~K}$ to 1300 at $5.587 \mathrm{GHz}$ and $77 \mathrm{~K}$. The shift in resonance frequency is shown in Fig. 3 where we plot normalised resonance frequency against temperature. The main contribution to the frequency shift arises from changes in penetration depth, which is a highly temperature dependent function, particularly just below $T_{C}$. The particular geometry of the resonator enhances the magnitude of this frequency shift.

The modelling of the change in $\mathrm{Q}$ and the frequency shift as a function of temperature is not trivial. The frequency shift is due almost entirely to the internal or kinetic inductance of the inductive element. Because the penetration depth increases with increasing temperature, the internal inductance increases and hence the frequency decreases. Simple expressions for the internal inductance 